\title{
A146 DECREASED SOLUBLE TUMOUR NECROSIS FACTOR EXPRESSION IN SPONDYLARTHRITIS VERSUS RHEUMATOID ARTHRITIS SYNOVITIS
}

N Yeremenko, C Ambarus, H Masdar, P P Tak, D L Baeten Clinical Immunology and Rheumatology, Academic Medical Center/University of Amsterdam, Amsterdam, The Netherlands

NY and CA contributed equally to this work

10.1136/ard.2010.129643w

Tumour necrosis factor (TNF) $\alpha$ is a pivotal mediator of synovial tissue inflammation in rheumatoid arthritis (RA) as well as in spondyloarthritis (SpA), as evidenced by the therapeutic effects of TNF blockade in both diseases. However, the levels of soluble TNF (sTNF) are strongly reduced in SpA versus RA synovial fluid (SF). Therefore we aimed to unravel the potential differences in TNF signalling between SpA and RA.

Synovial biopsies and SF were obtained from actively inflamed knee joints of RA and SpA patients. Soluble mediators in SF were analysed by ELISA. Synovial tissue expression was assessed by qPCR and by immunohistochemistry. Monocytes from peripheral blood of healthy donors were polarised in vitro and expression of transmembrane TNF (tmTNF) was measured by FACS. The production of sTNF by these polarised macrophage subsets was measured in the supernatant after lipopolysaccharide (LPS) stimulation.

The SF levels of sTNF were significantly lower in SpA than in RA ( $p<0.001)$. In contrast, however, mRNA levels of TNF were comparable between SpA and RA. This discrepancy could be explained by (1) increased intracellular degradation of TNF in SpA; (2) a relative increase of tmTNF vs sTNF in SpA; or (3) rapid extracellular clearance of sTNF by decoy receptors in SpA. As soluble TNF receptors can function as decoy 
receptors for sTNF, we investigated the expression of TNF-R1 and TNF-R2. Expression of TNF-R1 and TNF-R2 were moderately decreased at mRNA levels in SpA versus RA synovitis but were not different in the synovial tissue as assessed by immunohistochemistry. The levels of sTNF-R1 and sTNF-R2 in SF, however, were substantially lower in SpA than RA ( $p<0.001)$. To address a potential shift between tmTNF and sTNF, we next assessed the expression of the TNF-cleaving enzyme TACE. TACE mRNA levels in synovial tissue, as well as sIL-6R (also cleaved by TACE) and TIMP-3 (the inhibitor of TACE) levels in SF were similar in SpA and RA, suggesting that there is no difference in the expression/activity of TACE. Analysis of polarised macrophages revealed no difference between interferon (IFN) $\gamma$ (classically activated macrophages), interleukin (IL)4 (alternative activated macrophages) and IL10 (regulatory macrophages, a subset overrepresented in $\mathrm{SpA}$ ) polarised cells in terms of tmTNF expression. However, there was a suppression of sTNF secretion upon LPS activation by IL10 versus IFN $\gamma$ polarised macrophages. Western blotting on SpA and RA synovial tissue to assess the expression of tmTNF versus sTNF is currently being performed.

The differences in SF levels of sTNF between SpA and RA cannot be explained by an increased expression of its decoy receptors in SpA. Our data suggest that maintained membrane expression of both TNF receptors in combination with a decrease in the soluble decoy receptors might potentiate TNF signalling in SpA synovitis. It remains to be assessed whether this signalling is dependent on tmTNF rather than sTNF and how this impacts its downstream effects. 